\title{
Performance Analysis of an Integrated CS/PS Services CDMA System
}

\author{
Lin Wang, A. Hamid Aghvami, Fellow, IEEE, William G. Chambers, Jordi Pérez-Romero, Oriol Sallent, and \\ Mischa Dohler, Member, IEEE
}

\begin{abstract}
An analytical model is developed to investigate integrated CS/PS CDMA cellular systems with SIR-based power control. Because packet data are not very sensitive to delay, a defer-first-transmission transmission mode is usually applied to packet data transmission. In the mixed CS/PS environment, linklevel congestion control is designed to delay packet data, if necessary, to guarantee real-time delivery quality for CS traffic such as voice and CS data. In interference-limited CDMA cellular systems, because interference (both intracell and intercell) is the limit to spectral efficiency, congestion control is driven by the total received interference level at the BS. With congestion control at link level and SIR-based power control, the intracell and intercell interferences are closely related to each other. A recursive process is developed to evaluate the performance of such a CDMA system. Numerical results such as system capacity and data delay, both with and without power control error considered, are presented.
\end{abstract}

Index Terms-CDMA, cellular land mobile radio.

\section{INTRODUCTION}

$\mathbf{M}$ UCH research work has been conducted on the performance analysis of cellular CDMA systems. In [1], a framework based on interference analysis was developed. Further investigations [2]-[4] were carried out on the effects of power control error (PCE) on cellular CDMA capacity. More recent work [5], [6] offers a more accurate analysis taking into consideration specified power control schemes. However, the previous work is mainly concerned with conventional voiceonly CDMA systems.

Third-generation mobile systems such as UMTS are expected to offer equal strength for both circuit and packet switching (PS) services. The real-time high bit-rate data along with voice is still supposed to be supported by circuit switch, and non-realtime data services are supposed to use packet switch. In [2], Jansen et al. investigate the throughput of a packet CDMA cellular system, and further in [7], Manji et al. study a multimedia indoor packet CDMA system. However, in both researches, the same method was applied that was used for circuit switch-

Manuscript received June 27, 2003; revised August 24, 2004. This work was performed with the support of EPSRC grant No. GR/N64236/01 and IST EVEREST project (http://www.everest-ist.upc.es), which is partly funded by the European Community. The review of this paper was coordinated by Prof. B. Li.

L. Wang, A. H. Aghvami, W. G. Chambers, and M. Dohler are with the Center for Telecommunications Research, King's College London, WC2R 2LS, UK (e-mail: lin.wang@kcl.ac.uk; hamid.aghvami@kcl.ac.uk; bill.chambers@kcl. ac.uk; mischa.dohler@kcl.ac.uk).

J. Pérez-Romero and O. Sallent are with the Department of Telecommunication, Universitat Politècnica de Catalunya, Spain (e-mail: jorperez@ tsc.upc.edu; sallent@tsc.upc.edu).

Digital Object Identifier 10.1109/TVT.2005.851371 ing (CS) systems in [1]-[3]. Because most packet services use the defer-first-transmission (DFT) transmission mode associated with auto-repeat-request (ARQ) schemes, in congestion control state, congestion control function usually delays the PS data service to guarantee CS service quality.

In CDMA cellular systems, due to their interference-limited characteristic, the congestion control in each cell is usually designed to maintain the interference level below the maximum allowed interference level (MAIL) at BS (base station) for the uplink by control number of data users' transmission. To achieve this with the universal frequency reused CDMA cellular system, the congestion control is supposed to be a function of both intercell and intracell interference. With this function, a new user's transmission in one cell contributes to the intercell interference in other cells, so the congestion control in the other cells will act to control its own data users' transmission. This will then affect the interference in the original cell and so on. Thus, the intercell and intracell interference interact with each other through the congestion function. To study the effects of introducing packet transmission on voice capacity, the recursive model presented in [6], [8] is developed here to evaluate the integrated PS/CS environment so it can reflect the close interactive relationship of the intercell and intracell interference. Because PCE is a common problem in CDMA systems, we also study the impacts of PCE on the performance of the integrated CS/PS system. We develop this model further to make it suitable for investigating the system performance in the presence of PCE.

This article is structured as follows: In Section II, the system model is described. Section III presents the analytical models for both perfect power control and imperfect power control. Section IV contains numerical results. Finally, the conclusion is given in Section V.

\section{SYSTEM DESCRIPTION}

\section{A. Cellular Structure}

In this article, we consider a CDMA system consisting of equal-size hexagonal cells, as in Fig. 1. A BS is located at the center of each cell and employs directional antennas dividing its cell into three sectors. The shadow fading or slow fading is modeled as a log-normal distribution of $10^{\xi / 10}$, where $\xi$ is a Gaussian random variable with standard deviation $\sigma_{s}=8 \mathrm{~dB}$ and zero mean [1]. Hence, the effect of the path loss and shadow fading on the power gain is proportional to $d^{-4} 10^{\xi / 10}$, where $d$ is the distance from a user to the receiving cell site. All the users' signals are spread over the whole bandwidth with a constant chip rate $R_{c}$. Traffic is uniformly distributed. Furthermore, the time 


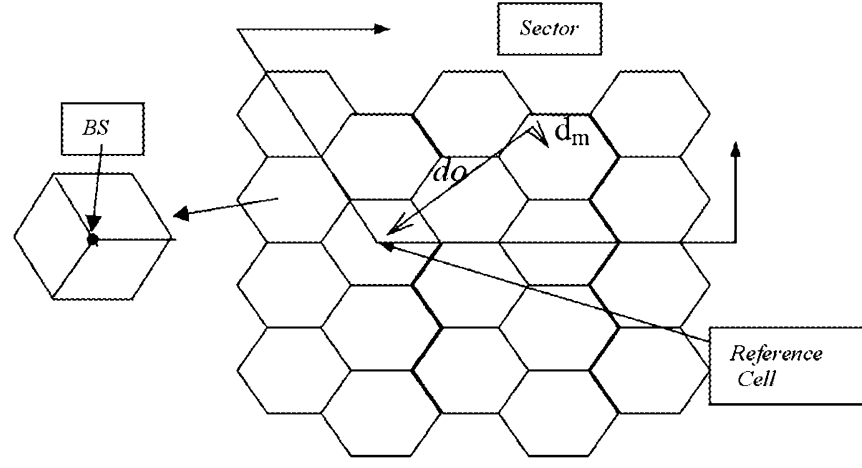

Fig. 1. Cellular structure.

axis is divided into frames with duration of $10 \mathrm{~ms}$ as in UMTS. Users are only allowed to start transmission at the beginning of a frame, and link-level control information such as scheduling is updated from frame to frame.

\section{B. Final Stage}

The MAIL $\left(I_{\max }\right)$ is limited at the BS receiver [9]. If the total received interference is more than this threshold, all the communications of the users in this cell are interrupted and outage occurs. The outage probability is defined as the probability of the total received interference at the BS receiver exceeding MAIL. It is given as

$$
P_{o} \equiv \operatorname{prob}\left(I_{\text {total }}>I_{\text {max }}\right)
$$

where $I_{\text {total }}$ is the total receiving power consisting of intercell interference $\left(I_{\text {inter }}\right)$, intracell interference $\left(I_{\text {intra }}\right)$, and background noise $(\eta)$.

Because there is no retransmission for CS services, it is necessary to keep the outage probability below $1 \%$ to provide a good quality of service [6]. Thus, we define the system capacity as the number of CS users that can be supported with a $1 \%$ outage probability. Here, SIR-based power control is applied to minimize the interference and achieve a better outage probability performance [6], [10]. In SIR-based power control systems, the power is adjusted according to the users' SIR requirements, so the power allocated to each user is expressed as

$$
S_{i}=\left(I_{\text {intra }}+I_{\text {inter }}+\eta\right) /\left(1+G_{i} / \gamma_{i}\right)
$$

where $G_{i}$ is the processing gain of user $i$ and $\gamma_{i}$ is the target SIR for user $i$.

If there are $k$ active users in the cell, the total intracell interference is given by summing (2):

$$
I_{\text {intra }}=\frac{\left(I_{\text {inter }}+\eta\right) \sum_{i=1}^{k}\left(G_{i} / \gamma_{i}+1\right)^{-1}}{1-\sum_{i=1}^{k}\left(G_{i} / \gamma_{i}+1\right)^{-1}} .
$$

\section{CS Transmission}

In this article, we consider two types of CS services defined in [11]: voice and long constrained delay (LCD) services. Due to the requirement of real-time delivery, CS is given high priority over the packet data service introduced later. An ON-OFF model is used to describe these two CS services' activity [1]. With

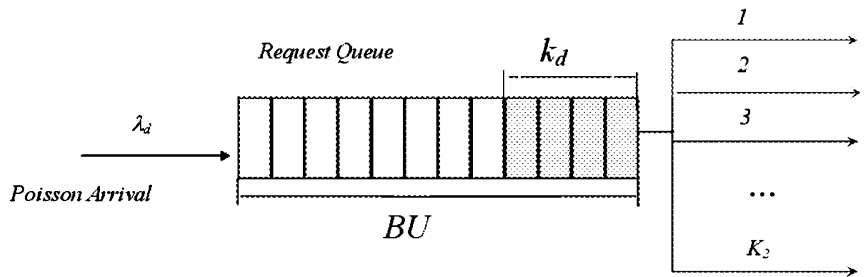

Fig. 2. PS data transmission.

activity factor $\alpha$, the activity random variable $\phi$ is given as

$$
\phi_{i}= \begin{cases}1 & \text { with probability of } \alpha_{i} \\ 0 & \text { otherwise }\end{cases}
$$

where $i$ is the service index (voice, LCD, and PS data).

\section{PS Data Transmission}

The data transmission model for the analytical model is shown in Fig. 2. Packet data messages are generated according a Poisson distribution with a mean value of $\lambda_{d}$ messages/s. Then the data messages are fragmented into smaller packets. Each packet just fits into one data block. Each message length is assumed to follow a geometric distribution with a mean value of $L$ packets. Once the message is generated, the packets will be stored in the user's buffer and a request will be sent to the BS through the random access channel. Then the request will be queued in the BS along with other users' requests in a request queue (Fig. 2). The user will wait for the scheduling transmission command from the BS. The request will be cleared after BS has completely received the message. In the $\mathrm{BS}$, only $B U$ requests can be queued. If the request queue is full, new requests will be rejected, and the data user is blocked. With the ARQ transmission scheme, if the transmission time is sufficient, an error-free communication can be achieved by retransmission diversity. Thus, performance measures such as BER and outage probability are not that important for packet data users compared with CS services. However, in packet data communication, the end users do experience queuing delay, so if a long delay occurs the queue will be filled up (i.e., the number of received requests is more than $B U$ in Fig. 2) and the new arrival requests will be blocked. Thus, new performance measures are introduced here to evaluate packet transmission performance: 1) average data delay, which is defined as the average time of a request stays in the BS request queue; and 2) the average blocking probability, which is defined as the ratio of the number of requests that are blocked to the total number of requests generated.

Because CDMA is deployed as a multiple access technique, a few users ( $K 2$ as shown in Fig. 2) are allowed to transmit their packets simultaneously. A value of $K 2$ is actually decided by the congestion function. In the mixed CS/PS environment, because CS services require real-time delivery, retransmission is impossible for CS services. Usually, a higher priority is given to CS services over PS services. Therefore, the congestion function is designed to schedule users' transmission of PS data and guarantee real-time delivery quality for CS data. As mentioned earlier in Section II-A, the total received interference in a BS receiver must not exceed a limit; otherwise, outage will occur. 
Thus, the main objective of congestion function is to maintain the total receiving power at BS below $I_{\max }$, by controlling the number of packet data users allowed to transmit.

With power constraint and with high priority given to CS data users, in interference limited CDMA systems, the number of simultaneously transmitting PS data users $(K 2)$ is a function (congestion function) of $k_{d}$ (the number of PS data requests queuing in the buffer, as shown in Fig. 2), $I_{\mathrm{CS}}$ (the interference contributed by intracell CS service users), and $I_{\text {inter }}$ (the intercell interference). It is given by

$$
\begin{aligned}
& K 2\left(k_{d}, I_{\mathrm{CS}}, I_{\text {inter }}\right) \\
& \quad=\left\lfloor\min \left(k_{d}, \max \left(0, \frac{T-I_{\mathrm{CS}}-I_{\text {inter }}-\eta}{P_{d}}\right)\right)\right\rfloor
\end{aligned}
$$

where $P_{d}$ represents the power allocated to single PS data users, $\lfloor x\rfloor$ means the maximum integer not greater than $x$, and $T$ is the control threshold, which is less than $I_{\max }$ and is a design parameter of the congestion control.

\section{E. Interactions Through Congestion Function}

From (5), it is found that $K 2$ also depends on intercell interference. So, the intercell and intracell interference interact with each other through the congestion function. For instance, if the congestion function for the reference cell in Fig. 1 were to allow an increase in the number of simultaneous transmitting users in the reference cell, the change would generate extra intercell interference affecting users in other cells. Following this change in the intercell interference, initially, in the other cells, the power for each user will be adjusted following (2). The congestion functions for those cells would update their values of $K 2$, taking changes in both intracell and intercell interference into account. This in turn would affect the intercell interference in the reference cell, and both power control and the congestion function in this cell would alter users' transmissions within its coverage. Then this would again affect the other cells' transmissions. However, a final steady state can be achieved as the interaction continues because of the existence of propagation attenuation. With the propagation attenuation, the intercell interference change caused by power control and congestion control in one BS will be reduced by propagation factor when it reaches the distant BS; thus, the distant BS will react to the reduced interference change, which will then affect the original $\mathrm{BS}$ with a further reduced change in intercell interference. Thus, as the interaction goes on, its effect on interference is gradually reduced to zero. To model this process, a recursive model similar to that in [6] is developed here to evaluate system performance of this integrated CS/PS cellular CDMA system.

\section{ANALYTICAL MODEL}

\section{A. Problem Formulation}

In the $t$ th, the system can be described by three state variables $k_{\mathrm{LCD}, t}, k_{\mathrm{vo}, t}$, and $k_{d, t}$. They are the number of active LCD users, the number of active voice users, and the number of queuing data users, respectively. A three-dimensional Markov chain can be used to model the integrated CS/PS system. Then at frame $t+1$, the state variables are given by

$$
\begin{aligned}
k_{\mathrm{LCD}, t+1} & =k_{\mathrm{LCD}, t}+a_{\mathrm{LCD}, t}-d_{\mathrm{LCD}, t} \\
k_{\mathrm{vo}, t+1} & =k_{\mathrm{vo}, t}+a_{\mathrm{vo}, t}-d_{\mathrm{vo}, t} \\
k_{d, t+1} & =\min \left(B U, k_{d, t}+a_{d, t}\right)-d_{d, t}
\end{aligned}
$$

where $a_{\mathrm{LCD}, t}$ and $a_{\mathrm{vo}, t}$ are the number of LCD users and voice users that become active, and $d_{\mathrm{LCD}, t}$ and $d_{\mathrm{vo}, t}$ are the number of LCD users and voice users that become silent in the $t$ th frame; $a_{d, t}$ is the number of new arrival data requests, and $d_{d, t}$ is the number of departing data users in $t$ th frame.

Note that, here, congestion function does not apply to CS services. So LCD and voice users' behavior depends completely on their own activity; however, due to the congestion function, PS data users' behavior depends on the CS service users and intercell interference. Then, the one-step transition probability can be expressed as

$$
\begin{aligned}
\underset{I_{t}}{\operatorname{Pr}}[ & \left.k_{\mathrm{LCD}, t+1}, k_{\mathrm{vo}, t+1}, k_{d, t+1} \mid k_{\mathrm{LCD}, t}, k_{\mathrm{vo}, t}, k_{d, t}\right] \\
= & \operatorname{Pr}\left[k_{\mathrm{LCD}, t+1} \mid k_{\mathrm{LCD}, t}\right] \operatorname{Pr}\left[k_{\mathrm{vo}, t+1} \mid k_{\mathrm{vo}, t}\right] \\
& \times \underset{I_{t}}{\operatorname{Pr}}\left[k_{d, t+1} \mid k_{d, t}, k_{\mathrm{vo}, t}, k_{\mathrm{LCD}, t}\right]
\end{aligned}
$$

where $I_{t}$ is the intercell interference at $t$ th frame.

The previous three-dimensional Markov process can be solved by three one-dimensional queues: a LCD queue with its steady-state probability denoted by $\pi_{k_{\mathrm{LCD}}}^{\mathrm{LCD}}$, and a voice queue with steady-state probability $\pi_{k_{\mathrm{vo}}}^{v}$, and a conditional PS data queue with its steady-state probability denoted by $\pi_{k_{d}}^{d}\left(k_{\mathrm{LCD}}, k_{\mathrm{vo}}, I_{\text {inter }}\right)$. According to Section II-C, $\pi_{k_{\mathrm{LCD}}}^{\mathrm{LCD}}$ and $\pi_{k_{\mathrm{vo}}}^{v}$ are given as

$$
\pi_{k_{\mathrm{LCD}}}^{\mathrm{LCD}}=\left(\begin{array}{c}
N_{\mathrm{LCD}} \\
k_{\mathrm{LCD}}
\end{array}\right) \alpha_{\mathrm{LCD}}^{k_{\mathrm{LCD}}}\left(1-\alpha_{\mathrm{LCD}}^{k_{\mathrm{LCD}}}\right)^{N_{\mathrm{LCD}}-k_{\mathrm{LCD}}}
$$

and

$$
\pi_{k_{\mathrm{vo}}}^{\mathrm{vo}}=\left(\begin{array}{c}
N_{\mathrm{vo}} \\
k_{\mathrm{vo}}
\end{array}\right) \alpha_{\mathrm{vo}}^{k_{\mathrm{vo}}}\left(1-\alpha_{\mathrm{vo}}\right)^{N_{\mathrm{vo}}-k_{\mathrm{vo}}} .
$$

If $k_{\mathrm{LCD}}, k_{\mathrm{vo}}$, and $I_{t}$ are given, subject to the condition of $0<k_{d}<=B U$, then $\pi_{k_{d}}^{d}\left(k_{\mathrm{LCD}}, k_{\mathrm{vo}}, I_{\mathrm{inter}}\right)$ can be obtained by solving a system of linear equations [12], with the transition probability $\operatorname{Pr}_{I_{t}}\left[k_{d, t+1} \mid k_{d, t}, k_{\mathrm{vo}, t}, k_{\mathrm{LCD}, t}\right]$. According to (6)(8), the transition probability for packet data users is given as

$$
\begin{aligned}
\underset{I_{t}}{\operatorname{Pr}}[ & \left.k_{d, t+1}=j \mid k_{d, t}=i, k_{\mathrm{LCD}, t}, k_{\mathrm{vo}, t}\right] \\
= & \sum_{a_{d, t}=0}^{\infty} A\left(a_{d, t}\right) B\left(\min \left(B U, a_{d, t}+i\right)\right. \\
& \left.-j, K_{2}\left(k_{\mathrm{LCD}, t}, k_{\mathrm{vo}, t}, I_{t}\right), \mu\right)
\end{aligned}
$$

where $A\left(a_{d, t}\right)$ denotes a Poisson arrival pattern that is given by $A(a)=\left(\lambda_{d}^{a} / a !\right) e^{-\lambda_{d}}$, and $K 2\left(k_{\mathrm{LCD}, t}, k_{\mathrm{vo}, t}, I_{t}\right)$ is the congestion function based on (5). With SIR-based power control, it is given as (Please see the equation at the bottom of next page.)

Moreover, $B(i, n, p)=\left(\begin{array}{c}n \\ j\end{array}\right) p^{i}(1-p)^{n-i}$ and $\mu \equiv(1-$ $\operatorname{PER}(\gamma)) / L$ is the average departure rate for the PS data at 
this frame, where PER is the packet error rate that is given by

$$
\mathrm{PER}=\sum_{i=m+1}^{d} B(i, d, \operatorname{BER}(\gamma))
$$

where $d$ is the packet size, $i$ is the number of error bits in the packet, and $m$ is the error correction capability.

With the knowledge of the instantaneous intercell interference $\left(I_{t}\right)$ and the number of active CS users $\left(k_{\mathrm{LCD}}, k_{\mathrm{vo}}\right)$, the conditional steady-state probability $\pi_{k_{d}}^{d}\left(k_{\mathrm{LCD}}, k_{\mathrm{vo}}, I_{\mathrm{inter}}\right)$ for data users can be derived from

$$
\begin{aligned}
& \sum_{i=0}^{B U} \pi_{i}^{d} \underset{I_{t}}{\operatorname{Pr}}\left(k_{d}=j \mid k_{d}=i\right)=\pi_{j}^{d} \\
& \sum_{i=0}^{B U} \pi_{i}^{d}=1 .
\end{aligned}
$$

Then the expressions for calculating performance measures, such as outage probability, average data delay, and data blocking probability, can be derived. Let $G(w)$ be the density function of intercell interference, which is still assumed to be a Gaussian distribution as in [6]. Then the outage probability defined in (1) is given as

$$
\begin{aligned}
P_{o}= & \operatorname{Pr}\left(I_{\text {inter }}+\rho+\eta>I_{\max }\right) \\
= & \int_{I_{\max }-\eta}^{\infty} G(w) d w \\
& +\int_{0}^{I_{\max }-\eta} \int_{I_{\max }-\eta}^{\infty} f_{\rho}(x) G(w) d w d x .
\end{aligned}
$$

To compute the average delay, first the average throughput $S$ needs to be derived; this is defined as the number of data packets successfully received from one PS data user per frame needs to be derived. With the congestion function (12), $S$ depends on the number of transmitting data users, which further depends on the number of transmitting CS users, intercell interference, and packet error rate, which is a function of SIR. Thus, the $S$ is given as

$$
\begin{gathered}
S \equiv \int_{0}^{\infty} \sum_{k_{\mathrm{LCD}}=0}^{N_{\mathrm{LCD}}} \sum_{k_{\mathrm{vo}}=0}^{N_{\mathrm{vo}}} \sum_{k_{d}=0}^{B U} \pi_{k_{\mathrm{vo}}}^{\mathrm{vo}} \pi_{k_{\mathrm{LCD}}}^{\mathrm{LCD}} \pi_{k_{d}}^{d}\left(k_{\mathrm{LCD}}, k_{\mathrm{vo}}, w\right) \\
\times K 2\left(k_{\mathrm{LCD}}, k_{\mathrm{vo}}, k_{d}, w\right)(1-\mathrm{PER}) G(w) d w
\end{gathered}
$$

where $K 2$ is given by (12).

Because SIR-based power control is applied, PER in this case is fixed. Thus, $K 2$ is the key factor to affect the data throughput. $K 2$ is subject to the value of $k_{\mathrm{LCD}}, k_{\mathrm{vo}}$, and $w$. With a fixed control threshold $T$, a higher CS traffic load (large values of $k_{\mathrm{LCD}}$ and $k_{\mathrm{vo}}$ ) will drive $K 2$ to a lower value and a lower throughput is expected and vice versa. The intercell interference $w$ also has the same effect on the throughput.

Then the average message delay, based on its definition, is given as (Please see the equation at the bottom of the page.) where the integral above the dominator represents the average traffic load in terms of the total number of packets in the buffer.

The blocking probability is given as

$$
\begin{aligned}
P_{b}= & \sum_{d=1}^{\infty} \int_{0}^{\infty} \sum_{k_{\mathrm{LCD}}=0}^{N_{\mathrm{LCD}}} \sum_{k_{\mathrm{vo}}=0}^{N_{\mathrm{vo}}} \sum_{k_{d}=0}^{B U} A(d) \\
& \times \pi_{k_{\mathrm{LCD}}}^{\mathrm{LCD}} \pi_{k_{\mathrm{vo}}}^{\mathrm{vo}} \pi_{k_{d}}^{d}\left(k_{\mathrm{vo}}, k_{\mathrm{LCD}}, w\right) \\
& \times\left(\frac{\max \left(0, d+k_{d}-B U\right)}{d}\right) G(w) d w .
\end{aligned}
$$

From (15) to (17), it is found that, if intercell interference is determined, the steady-state probability of the systems will be obtained, and then the performance measures can be computed from (15) to (17). In general, a larger intercell interference or a higher load of CS traffic will increase the number of packets in the buffer and reduce the throughput as shown in (15); thus, a longer delay and a larger blocking probability are expected.

\section{B. Analytical Model With Perfect Power Control}

In this section, an analytical model is developed to deal with a system with perfect power control. In perfect power control, the received $\operatorname{SIR}(\gamma)$ for each user is a constant. To keep the total interference below $I_{\max }$, the number of transmitting data users must satisfy the following condition:

$$
k_{\mathrm{LCD}} S_{\mathrm{LCD}}+k_{\mathrm{vo}} S_{\mathrm{vo}}+K_{2} S_{d}+\eta+I_{\mathrm{inter}} \leq I_{\max }
$$

where the signal power $S$ for each type of user is given by (1).

A procedure similar to that in [6] shows that the mean value and variance of the intercell interference are given as

$$
m_{I}=M\left(\mu, \sigma_{s}\right) E[\rho]
$$

and

$$
\sigma_{I}^{2} \approx A\left(\mu, \sigma_{s}\right) E\left[\rho^{2}\right]-B\left(\mu, \sigma_{s}\right) E^{2}[\rho]
$$

where

$$
M\left(\mu, \sigma_{s}\right)=\iint_{A}\left(\frac{d_{m}}{d_{o}}\right)^{4} f\left(\frac{d_{m}}{d_{o}}\right) \frac{2}{3 \sqrt{3}} d A
$$

$$
\begin{gathered}
K 2\left(k_{\mathrm{LCD}}, k_{\mathrm{vo}}, k_{d}, I_{t}\right)=\left\lfloor\max \left(0, \min \left(k_{d}, \frac{T\left(1-\left(\frac{k_{\mathrm{LCD}}}{C_{\mathrm{LCD}}}+\frac{k_{\mathrm{vo}}}{C_{\mathrm{vo}}}\right)\right)-I_{t}-\eta}{T\left(\frac{1}{C_{d}}\right)}\right)\right)\right\rfloor \\
D=\frac{\int_{0}^{\infty} \sum_{k_{\mathrm{LCD}}=0}^{N_{\mathrm{LCD}}} \sum_{k_{\mathrm{vo}}=0}^{N_{\mathrm{vo}}} \sum_{k_{d}=0}^{B U} \pi_{k_{\mathrm{vo}}}^{\mathrm{vo}} \pi_{k_{\mathrm{LCD}}}^{\mathrm{LCD}} \pi_{k_{d}}^{d}\left(k_{\mathrm{vo}}, k_{\mathrm{LCD}}, w\right) \cdot k_{d} \cdot L \cdot G(w) d w}{S}
\end{gathered}
$$




$$
\begin{aligned}
A\left(\mu, \sigma_{s}\right)= & \iint_{A}\left(\frac{d_{m}}{d_{o}}\right)^{2 \mu} \exp \left[\left(\frac{\sigma_{s} \ln 10}{5}\right)^{2}\right] \\
& \times\left\{1-Q\left[\frac{10 \mu}{\sqrt{2 \sigma_{s}^{2}}} \log _{10}\left(\frac{d_{m}}{d_{o}}\right)\right.\right. \\
& \left.\left.-\sqrt{2 \sigma_{s}^{2}}\left(\frac{\ln 10}{5}\right)\right]\right\} d A
\end{aligned}
$$

and

$$
\begin{aligned}
B\left(\mu, \sigma_{s}\right)= & \iint_{A}\left(\frac{d_{m}}{d_{o}}\right)^{2 \mu} \exp \left[2\left(\frac{\sigma_{s} \ln 10}{10}\right)^{2}\right] \\
& \times\left\{1-Q\left[\frac{10 \mu}{\sqrt{2 \sigma_{s}^{2}}} \log _{10}\left(\frac{d_{m}}{d_{o}}\right)\right.\right. \\
& \left.\left.-\sqrt{2 \sigma_{s}^{2}} \frac{\ln 10}{10}\right]\right\}^{2} d A .
\end{aligned}
$$

With the Markov chain model, we have

$$
\begin{aligned}
E[\rho]= & \int_{0}^{\infty} \sum_{k_{\mathrm{LCD}}=0}^{N_{\mathrm{LCD}}} \sum_{k_{\mathrm{vo}}=0}^{N_{\mathrm{vo}}} \sum_{k_{d}=0}^{B U} \pi_{k_{\mathrm{vo}}}^{\mathrm{vo}} \pi_{k_{\mathrm{LCD}}}^{\mathrm{LCD}} \pi_{k_{d}}^{d} I_{\text {intra }} \\
& \cdot G(w) d w
\end{aligned}
$$

and

$$
\begin{aligned}
E\left[\rho^{2}\right]= & \int_{0}^{\infty} \sum_{k_{\mathrm{LCD}}=0}^{N_{\mathrm{LCD}}} \sum_{k_{\mathrm{vo}}=0}^{N_{\mathrm{vo}}} \sum_{k_{d}=0}^{B U} \pi_{k_{\mathrm{vo}}}^{\mathrm{vo}} \pi_{k_{\mathrm{LCD}}}^{\mathrm{LCD}} \pi_{k_{d}}^{d} I_{\text {intra }}^{2} \\
& \cdot G(w) d w
\end{aligned}
$$

where $\pi_{k_{\mathrm{vo}}}^{\mathrm{vo}}$ and $\pi_{k_{\mathrm{LCD}}}^{\mathrm{LCD}}$ are computed from (9) and (10) directly, $\pi_{k_{d}}^{d}$ is computed by (13) with a given intercell interference value, $\rho$ represents the total intracell interference, and $I_{\text {intra }}$ is given by (3).

With congestion control and SIR-based power control from the previous description, intracell interference and intercell interference are closely related to each other. This makes it impossible to compute the intercell interference in a straightforward manner as in [1], [2], and [7]. So a recursive process similar to that in [6] is presented here to compute the mean value $m_{I}$ and variance $\sigma_{I}^{2}$ of the intercell interference as follows:

1) Set $I_{\text {inter }}, m_{I}$, and $\sigma_{I}^{2}$ to 0 , and calculate $I_{\text {intra }}$ according to (3);

2) Calculate $E[\rho]$ and $E\left[\rho^{2}\right]$ from (21) and (22);

3) Calculate $m_{I}$ and $\sigma_{I}^{2}$ from (19) and (20);

4) Repeat steps 2 and 3 until $m_{I}$ and $\sigma_{I}^{2}$ have convergence. ${ }^{1}$

After $m_{I}$ and $\sigma_{I}^{2}$ are determined, the outage probability for perfect power control can be computed as follows:

$$
P_{o}=\operatorname{Pr}\left(I_{\text {inter }}+\rho+\eta>I_{\max }\right)
$$

\footnotetext{
${ }^{1}$ Due to the attenuation of the propagation channel, the change of interference decreases every repeating step in the recursive process; thus, a convergence can be guaranteed in this calculation.
}

$$
\begin{aligned}
= & \int_{I_{\max }-\eta_{o}}^{\infty} G(w) d w \\
& +\int_{0}^{I_{\max }-\eta} \int_{I_{\max }-\eta-w}^{\infty} f_{\rho}(x) G(w) d x d w \\
= & \int_{I_{\max }-\eta}^{\infty} G(w) d w \\
& +\sum_{k_{\mathrm{LCD}}=0}^{N_{\mathrm{LCD}}} \sum_{k_{\mathrm{vo}}}^{N_{\mathrm{vo}}} \pi_{k_{\mathrm{LCD}}}^{\mathrm{LCD}} \pi_{k_{\mathrm{vo}}}^{\mathrm{vo}} Q \\
& \times\left(\frac{I_{\max }\left(1-\frac{k_{\mathrm{LCD}}}{C_{\mathrm{LCD}}}-\frac{k_{\mathrm{vo}}}{C_{\mathrm{vo}}}\right)-\eta-m_{I}}{\sigma_{I}}\right)
\end{aligned}
$$

In the perfect power control case, with congestion control, PS transmission is strictly controlled by the congestion control function. So CS and intercell interference are the main reasons for outage.

Then with $m_{I}$ and $\sigma_{I}^{2}$, PS data users' performance, such as throughput, delay, and blocking probability, can be calculated directly from (15) to (17).

\section{Analytical Model With Imperfect Power Control}

In SIR-based power control, PCE causes variations in received SIR. With PCE, the received SIR actually follows a log-normal distribution [9]. So a lognormal random variable $\Gamma_{i}=10^{\Omega i / 10}$ is used here to model the $i$ th user's received SIR at the BS. Here, $\Omega_{i}$ is a Gaussian random variable with a mean $\mu_{\Omega i} \mathrm{~dB}$ (the target SIR) and standard deviation $\sigma_{e} \mathrm{~dB}$ (the PCE). Because the same power control algorithm is applied to all users, the PCE is assumed to be the same for all. Then (3) is rewritten as

$$
I_{\text {intra }}\left(k, I_{\text {inter }}\right)=\frac{\left(I_{\text {inter }}+\eta\right) \sum_{i=1}^{k}\left(G_{i} / \Gamma_{i}+1\right)^{-1}}{1-\sum_{i=1}^{k}\left(G_{i} / \Gamma_{i}+1\right)^{-1}} .
$$

Now, let $Z=\sum_{i=1}^{k}\left(G_{i} / \Gamma_{i}+1\right)^{-1}$. Then,

$$
I_{\text {intra }}\left(Z, I_{\text {inter }}\right)=\frac{\left(I_{\text {inter }}+\eta\right) Z}{1-Z}
$$

with an approximation of $Z$ as a $\log$-normal distribution, as presented in the Appendix.

Then the outage probability becomes

$$
\begin{aligned}
P_{o}= & \operatorname{prob}\left(\mathrm{Z}>\left(I_{\max }-I_{\text {inter }}-\eta\right) / I_{\max }\right) \\
= & \operatorname{prob}\left(e^{X_{z}}>\left(I_{\max }-I_{\text {inter }}-\eta\right) / I_{\max }\right) \\
= & \operatorname{prob}\left(X_{z}>\ln \left(\left(I_{\max }-I_{\text {inter }}-\eta\right) / I_{\max }\right) \mid I_{\max }\right. \\
& \left.-\eta>I_{\text {inter }}\right) \\
& +\operatorname{prob}\left(I_{\text {inter }}>I_{\max }-\eta\right) .
\end{aligned}
$$

So, at each frame $t$, if the traffic load in terms of the number of users is known, the outage probability, in the presence of $k$ 
active users, is given as

$$
\begin{array}{r}
P_{o, t}(k)=\int_{0}^{I_{\max }-\eta} Q\left(\frac{\ln \left(\frac{I_{\max }-w-\eta}{I_{\max }}\right)-m_{z}(k)}{\sigma_{z}(k)}\right) G(w) d w \\
+\int_{I_{\max }-\eta}^{\infty} G(w) d w . \quad \text { (27) }
\end{array}
$$

Note that in the mixed traffic environment, $m_{z}(k)$ and $\sigma_{z}(k)$ are functions of $k_{\mathrm{LCD}}, k_{\mathrm{vo}}$, and the number of transmitting data users $K 2$. So, from now on, $m_{z}(k)$ and $\sigma_{z}(k)$ are replaced by $m_{z}\left(k_{\mathrm{LCD}}, k_{\mathrm{vo}}, K 2\right)$ and $\sigma_{z}\left(k_{\mathrm{LCD}}, k_{\mathrm{vo}}, K 2\right)$.

In the presence of PCE, even with congestion control, the outage probability is also affected by intracell interference. In this case, with the same congestion function, the departure rate of packet data users is given by (Please see the equation at the bottom of the page.)

With the Markov chain model presented in Section III-A, the steady-state probabilities can be derived only after replacing the departure rate with the previous expression. Then the complete outage probability is given by (Please see the equation at the bottom of the page.)

So, to derive all the performance measures from (15) to (17), intercell interference is now the only random variable still to be determined. Following the same procedure, $E[\rho]$ and $E\left[\rho^{2}\right]$ are given as

$$
\begin{aligned}
E[\rho]= & \sum_{k_{\mathrm{vo}}=0}^{N_{\mathrm{vo}}} \sum_{k_{\mathrm{LCD}}=0}^{N_{\mathrm{LCD}}} \int_{0}^{\infty} \int_{0}^{\infty} \sum_{k_{d}=0}^{B U} \pi_{k_{\mathrm{vo}}}^{\mathrm{vo}} \pi_{k_{\mathrm{LCD}}}^{\mathrm{LCD}} \pi_{k_{d}}^{d} \\
& \times I_{\text {intra }}(z, w) f_{z}(z) G(w) d z d w
\end{aligned}
$$

and

$$
\begin{aligned}
E\left[\rho^{2}\right]= & \sum_{k_{\mathrm{vo}}=0}^{N_{\mathrm{vo}}} \sum_{k_{\mathrm{LCD}}=0}^{N_{\mathrm{LCD}}} \int_{0}^{\infty} \int_{0}^{\infty} \sum_{k_{d}=0}^{B U} \pi_{k_{\mathrm{vo}}}^{\mathrm{vo}} \pi_{k_{\mathrm{LCD}}}^{\mathrm{LCD}} \pi_{k_{d}}^{d} \\
& \times I_{\text {intra }}^{2}(z, \varpi) f_{z}(z) G(w) d z d w
\end{aligned}
$$

where $f_{z}(z)$ is the pdf of $Z$.

So repeat the recursive procedure as follows:

1) Set $m_{I}$ and $\sigma_{I}$ to zero, and calculate $E[\rho]$ and $E\left[\rho^{2}\right]$ from (29) and (30);
2) Calculate $m_{I}$ and $\sigma_{I}^{2}$ from (19) and (20);

3) Calculate $E[\rho]$ and $E\left[\rho^{2}\right]$ from (29) and (30);

4) Calculate $m_{I}$ and $\sigma_{I}^{2}$ from (19) and (20);

5) Repeat steps 3 and 4 until $m_{I}$ and $\sigma_{I}^{2}$ have convergence. In the presence of PCE, the throughput is given as

$$
\begin{aligned}
S= & \sum_{k_{\mathrm{vo}}=0}^{N_{\mathrm{vo}}} \sum_{k_{\mathrm{LCD}}=0}^{N_{\mathrm{LCD}}} \int_{0}^{\infty} \sum_{k_{d}=0}^{B U} \pi_{k_{\mathrm{vo}}}^{\mathrm{vo}} \pi_{k_{\mathrm{LCD}}}^{\mathrm{LCD}} \pi_{k_{d}}^{d} \\
& \times K 2\left(k_{\mathrm{LCD}}, k_{\mathrm{vo}}, k_{d}, w\right)(1-\operatorname{PER}(\gamma d)) \\
& \times\left(1-P_{o, t}\left(k_{\mathrm{LCD}}, k_{\mathrm{vo}}, k_{d}\right)\right) G(w) d w
\end{aligned}
$$

and the delay is given as (Please see the equation at the bottom of the page.) The blocking probability is

$$
\begin{aligned}
P_{b}= & \sum_{d=1}^{\infty} \int_{0}^{\infty} \sum_{k_{\mathrm{LCD}}=0}^{N_{\mathrm{LCD}}} \sum_{k_{\mathrm{vo}}=0}^{N_{\mathrm{vo}}} \sum_{k_{d}=0}^{B U} A(d) \\
& \times \pi_{k_{\mathrm{LCD}}}^{\mathrm{LCD}} \pi_{k_{\mathrm{vo}}}^{\mathrm{vo}} \pi_{k_{d}}^{d}\left(k_{\mathrm{vo}}, k_{\mathrm{LCD}}, w\right) \\
& \times\left(\frac{\max \left(0, d+k_{d}-B U\right)}{d}\right) G(w) d w .
\end{aligned}
$$

\section{DISCUSSION AND NUMERICAL RESUlTS}

In this section, the numerical results obtained through the previous analytical models are presented, which mainly focus on the CS capacity, effects of PS service on CS capacity, and PS performance with PCE. Simulations are performed with IST EVEREST simulators [13] to verify the mean and variance of intercell interference derived by the analytical model. We found the errors are limited within $1 \%$ with perfect power control and within $2 \%$ in the presence of PCEs.

\section{A. CS Services Capacity}

SIR-based power-controlled system capacity has been discussed in detail in [6] and [8]. The results presented in this section are somewhat different. They emphasize the effects of PCE and the restriction on total received power. Neither of these issues was studied in [6] and [8]. The system parameters used here for the numerical evaluation are given in Table I. Most of the parameters are chosen based on the $3 \mathrm{GPP}$ specifications. In

$$
\begin{aligned}
\mu & =\frac{\left(1-\operatorname{PER}\left(\gamma_{d}\right)\right)\left(1-P_{o, t}\left(k_{\mathrm{LCD}}, k_{\mathrm{vo}}, K_{2}\left(k_{\mathrm{LCD}}, k_{\mathrm{vo}}, k_{d}, I_{t}\right)\right)\right)}{L} . \\
P_{o}= & \sum_{k_{\mathrm{LCD}}=0}^{N_{\mathrm{LCD}}} \sum_{k_{\mathrm{vo}}=0}^{N_{\mathrm{vo}}} \pi_{k_{\mathrm{LCD}}}^{\mathrm{LCD}} \pi_{k_{\mathrm{vo}}}^{\mathrm{vo}} \int_{0}^{I_{\mathrm{max}}-\eta} \sum_{k_{d}=0}^{B U} \pi_{k_{d}}^{d} \times Q\left(\frac{\ln \left(\frac{I_{\max }-w-\eta}{I_{\max }}\right)-m_{z}\left(k_{\mathrm{LCD}}, k_{\mathrm{vo}}, K 2\left(k_{\mathrm{LCD}}, k_{\mathrm{vo}}, w\right)\right)}{\sigma_{z}\left(k_{\mathrm{LCD}}, k_{\mathrm{vo}}, K 2\left(k_{\mathrm{LCD}}, k_{\mathrm{vo}}, w\right)\right)}\right) G(w) d w \\
& +\int_{I_{\max }-\eta}^{\infty} G(w) d w .
\end{aligned}
$$

$$
D=\frac{\sum_{k_{\mathrm{vo}}=0}^{N_{\mathrm{o}}} \sum_{k_{\mathrm{LCD}}=0}^{N_{\mathrm{LCD}}} \int_{0}^{\infty} \sum_{k_{d}=0}^{B U} \pi_{k_{\mathrm{vo}}}^{\mathrm{vo}} \pi_{k_{\mathrm{LCD}}}^{\mathrm{LCD}} \pi_{k_{d}}^{d} k_{d} L G(w) d w}{S}
$$


TABLE I

SYSTEM PARAMETERS

\begin{tabular}{l|l}
\hline \hline Total Bandwidth & $5 \mathrm{MHz}$ \\
\hline Voice Information Rate & $8 \mathrm{~kb} / \mathrm{s}$ \\
\hline Voice Transmission Rate & $30 \mathrm{~kb} / \mathrm{s}$ \\
\hline Voice Activity Factor & $3 / 8$ \\
\hline Voice $\mathrm{E}_{\mathrm{b}} / \mathrm{N}_{\mathrm{o}}$ requirement & $3.3 \mathrm{~dB}$ \\
\hline LCD information rate & $60 \mathrm{~kb} / \mathrm{s}$ \\
\hline LCD transmission rate & $240 \mathrm{~kb} / \mathrm{s}$ \\
\hline LCD $\mathrm{E}_{\mathrm{b}} / \mathrm{N}_{\circ}$ requirement & $2.4 \mathrm{~dB}$ \\
\hline PS Data Rate & $64 \mathrm{~kb} / \mathrm{s}$ \\
\hline PS $\mathrm{E}_{\mathrm{b}} / \mathrm{N}_{\mathrm{o}}$ requirement & $2.0 \mathrm{~dB}$ \\
\hline$\eta$ & $0 \mathrm{~dB}$ \\
\hline \hline
\end{tabular}

TABLE II

SingLE-CELL VOICE CAPACITY

\begin{tabular}{c|c|c|c}
\hline Imax(dB) & 6 & 10 & 15 \\
\hline PCE $(\mathrm{dB})$ & & & \\
\hline 0 & 92 & 113 & 122 \\
\hline 1 & 88 & 109 & 118 \\
\hline 2 & 77 & 96 & 104 \\
\hline 3 & 60 & 75 & 83 \\
\hline \hline
\end{tabular}

TABLE III

Multicell Voice CAPACITY

\begin{tabular}{c|c|c|c}
\hline Imax $(\mathrm{dB})$ & 6 & 10 & 15 \\
\cline { 1 - 3 } & & & \\
\hline 1 & $57(38 \%)$ & $76(32.7 \%)$ & $87(28.7 \%)$ \\
\hline 2 & $55(37.5 \%)$ & $74(32.1 \%)$ & $85(28 \%)$ \\
\hline 3 & $50(35 \%)$ & $67(30 \%)$ & $76(26.9 \%)$ \\
\hline \hline
\end{tabular}

this capacity evaluation, single-cell capacity is studied first as a benchmark for further evaluation in the multicell environment. An outage probability of $1 \%$ is chosen as the requirement for all CS services [6]. The single cell voice capacities with different power constraints and PCEs are given in Tables II and III. As expected, voice capacity increases as the power constraint increases; however, this increase becomes less and less significant with increasing MAIL, regardless of whether PCE exists. Also, with any given MAIL values in both single-cell and multicell situations, the capacity decreases as the PCE increases. It is also easy to see that the rate of voice capacity reduction due to PCE becomes larger because of the increase in the variance of the random variable $Z$ (Section III-C).

In Table III, the numbers shown as percentages are the capacity loss due to multicell interference. For any given MAIL (e.g., $6 \mathrm{~dB}$ ), as the PCE increases, the capacity loss decreases; for example, the capacity loss due to multicell interference decreases from $38 \%$ for $\mathrm{PCE}=0 \mathrm{~dB}$ to $30 \%$ for $\mathrm{PCE}=3 \mathrm{~dB}$. The rate of decrease in the capacity loss increases as PCE increases. Thus, from $\mathrm{PCE}=0$ to $\mathrm{PCE}=1 \mathrm{~dB}$ the capacity loss changes from $38 \%$ to $37.5 \%$, whereas from $\mathrm{PCE}=2 \mathrm{~dB}$ to $\mathrm{PCE}=3 \mathrm{~dB}$ the capacity loss changes from $35 \%$ to $30 \%$. This is mainly because, as PCE increases, the outage probability caused by PCE becomes increasingly dominant in the total outage events. Table

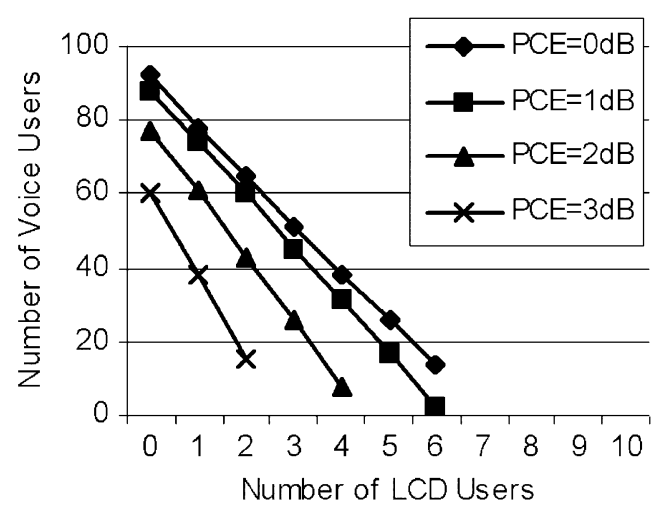

(a)

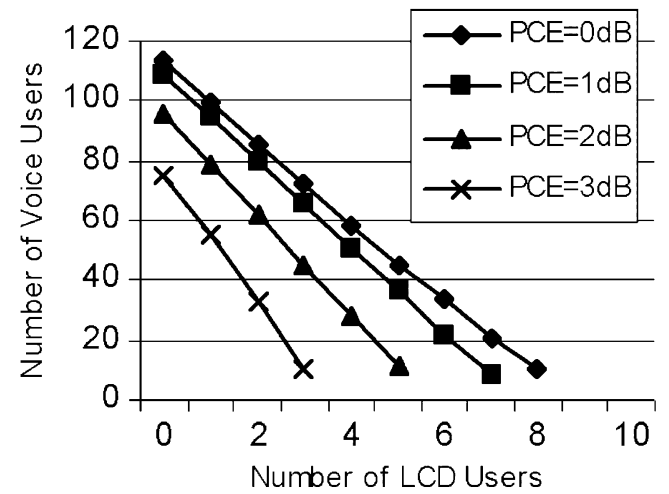

(b)

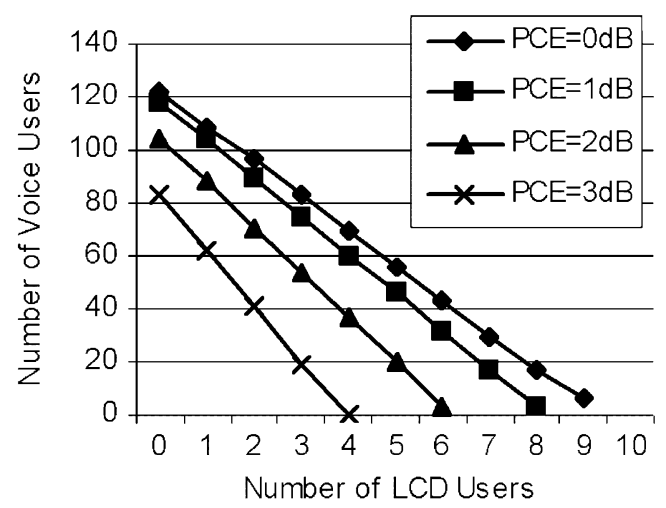

(c)

Fig. 3. Single-cell voice/lcd capacity boundaries: (a) $I_{\max }=6 \mathrm{~dB}$; (b) $I_{\max }=10 \mathrm{~dB}$; (c) $I_{\max }=15 \mathrm{~dB}$.

III also gives the effects of MAIL on the capacity loss due to multicell interference. Basically, the capacity loss decreases as MAIL increases, because, with larger MAIL, more users can be accommodated, which helps smooth out the variance.

Figs. 3 and 4 give the integrated LCD/voice capacity boundaries for single- and multicell situations, respectively. The linear relationship of the LCD and voice is there with various MAIL values. The voice capacity loss rate caused by adding LCD users increases as the PCE increases. In Figs. 3 and 4, the areas under the curves represent the valid capacity for the dual-service system for an outage probability of $1 \%$. The calculated capacity loss based on this is shown in Table IV. Basically, it has the same trend as the single voice service. 


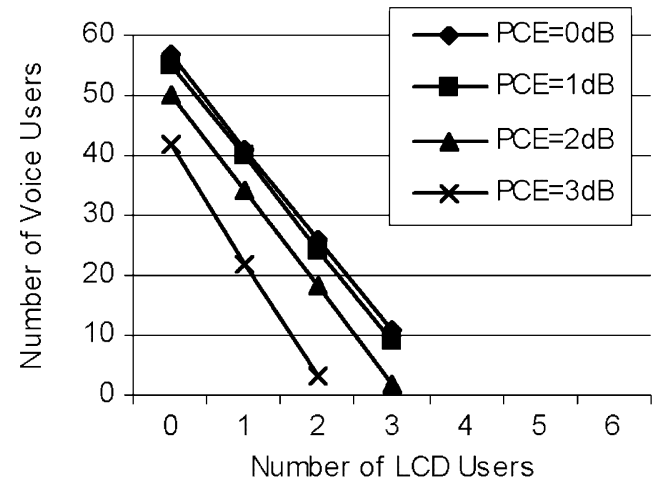

(a)

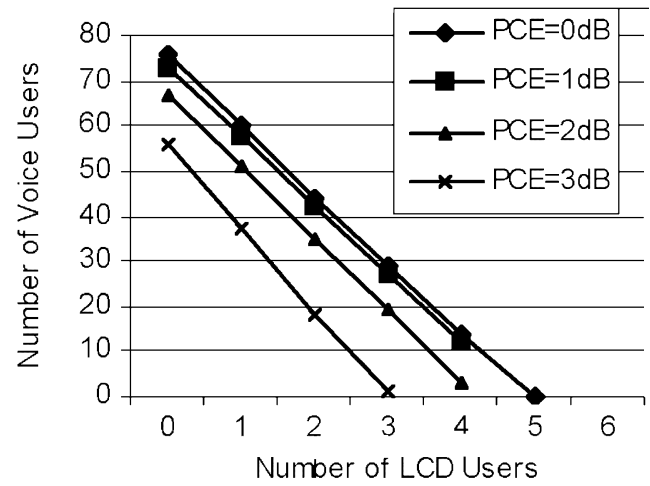

(b)

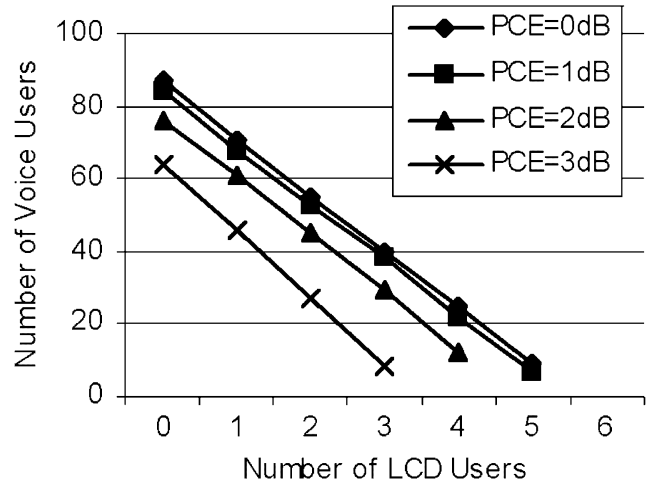

(c)

Fig. 4. Multi-cell voice/LCD capacity boundaries: (a) $I_{\max }=6 \mathrm{~dB}$; (b) $I_{\max }=10 \mathrm{~dB}$; (c) $I_{\max }=15 \mathrm{~dB}$.

\section{B. PS Data Impacts on Voice Capacity}

In this section, the results focus on effects of PS data on voice capacity. In this numerical evaluation, half-rate convolution coding is applied as inner coding and BCH coding is applied as outer coding to cooperate with a selective ARQ scheme for data users. A BCH $(255240,5)$ code is used for packet data transmissions, and the basic channel rate for PS data is $60 \mathrm{~kb} / \mathrm{s}$. (The spreading factor is 64.) With these parameters, a portion of each frame has been left for ARQ signaling transmission. With this coding scheme, the received SIR at the BS for a PS data transmission should be kept close to $2 \mathrm{~dB}$ to achieve a maximum throughput in CDMA packet radio systems [14]. With congestion function (5), the control threshold $T$ actually decides the resource allocation between CS and PS service. Thus, our evaluation first exams
TABLE IV

MulTiSERVICE CAPACITY LOSS

\begin{tabular}{l|l|l|l}
\hline \hline \multicolumn{1}{|l|}{$\operatorname{Imax}(\mathrm{dB})$} & 6 & 10 & 15 \\
$\mathrm{PCE}(\mathrm{dB})$ & & & \\
\hline 0 & $67.8 \%$ & $61.7 \%$ & $60.6 \%$ \\
\hline 1 & $64.2 \%$ & $58.7 \%$ & $55.1 \%$ \\
\hline 2 & $54.7 \%$ & $48.9 \%$ & $44.9 \%$ \\
\hline 3 & $49.7 \%$ & $34.2 \%$ & $32.9 \%$ \\
\hline \hline
\end{tabular}

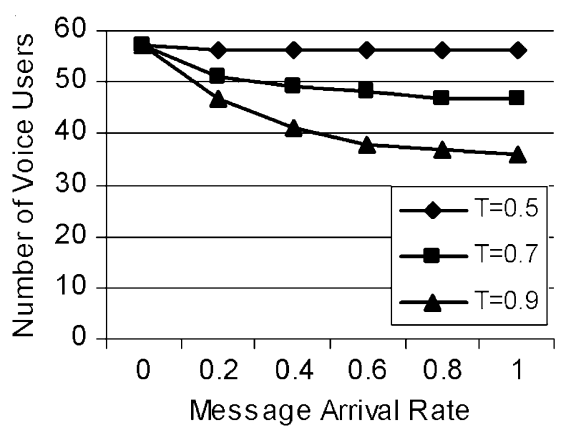

Fig. 5. Effects of PS data on voice capacity with various T values.

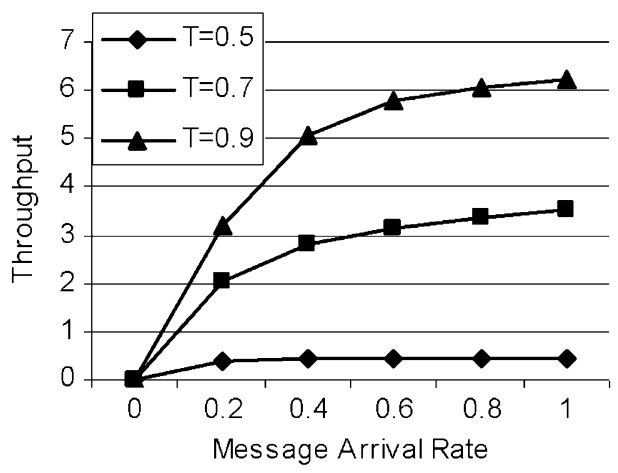

Fig. 6. Data throughput with various T values.

the effects of data traffic load on the voice capacity with various $T$ values as shown in Fig. 5. In Fig. 5, with a larger $T$ such as $0.9\left(T=0.9 I_{\max }\right)$, the reduction in voice capacity caused by data transmission is more significant and with a smaller $T$, such as 0.5 , the voice capacity is hardly affected by data traffic load. As mentioned previously, with congestion control function (5), the threshold $T$ actually decides the tradeoff between voice and data performance. With a larger $T$, data traffic is able to take more resource, a higher throughput is achieved for data users as shown in Fig. 6, and fewer voice users can be supported in the system, and vice versa. Also in Fig. 5, with a certain $T$ value, the voice capacity reduction becomes increasingly less as the data traffic load increases. For example, with $T=0.7$, the voice capacity is reduced from 57 to 51 as the message arrival rate increases from 0 to 0.2 , whereas the voice capacity is almost the same as the message arrival rate increases from 0.8 to 1 . This is mainly because, as data traffic load increases, the resources used by data user are getting closer and closer to the threshold $T$; thus, it becomes increasingly more difficult to increase data throughput due to congestion control, as shown in Fig. 6. In this, the increase in interference (both intercell and 


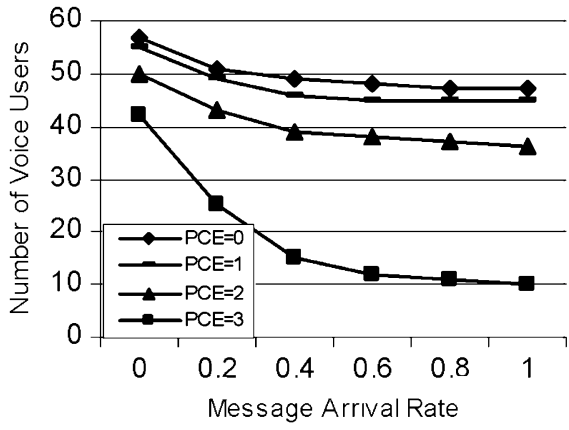

Fig. 7. Effects of PS data on voice capacity with various PCEs.

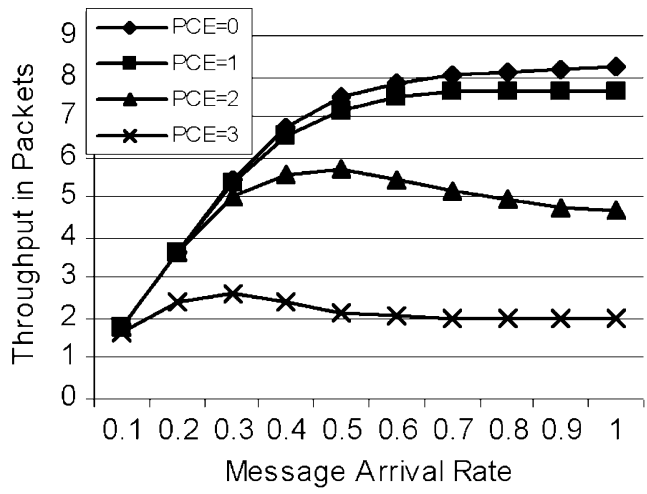

Fig. 8. Effect of PCE on PS throughput (1 LCD, 20 voice).

intracell interference) caused by data users is getting less and less, so the capacity reduction is also getting less and less. This indicates that the operators should adjust the $T$ value based on the traffic composition of the systems to achieve an optimum operation point in terms of total users' (both CS and PS) quality of service satisfaction.

Fig. 7 shows effects of PS data on the voice capacity with various PCEs. In Fig. 7, with a fixed PCE, the curve follows a similar trend as those without PCE (as shown in Fig. 5) (i.e., the capacity loss is getting less as data traffic load is increasing). As PCE increases, the voice capacity loss due to the introduction of data increases, and this increase is getting larger and larger. For example, as data increase from 0.2 to 0.4 with $1 \mathrm{~dB}$ PCE, the voice capacity decreases from 50 to 46 , with a deduction of $8 \%$, whereas with $3 \mathrm{~dB}$ PCE, the voice capacity decreases from 25 to 15 with a deduction of $40 \%$. So with larger PCE, a smaller $T$ value would favor voice capacity. Providing the linear relationship of voice and LCD capacity when adding LCD, the system performance is quite similar.

\section{Effects of PCE on Data Performance}

Fig. 8 shows the throughput with various PCEs. As expected, with PCE increasing, the throughput is reduced, and this actually leads to an increase in message delay and blocking probability as shown in Figs. 9 and 10. The is mainly because, as the PCE increases, more outage events occur as shown in Fig. 11, and thus, a lower throughput is expected. Results in the last section show that as PCE increases, CS capacity decreases more rapidly.

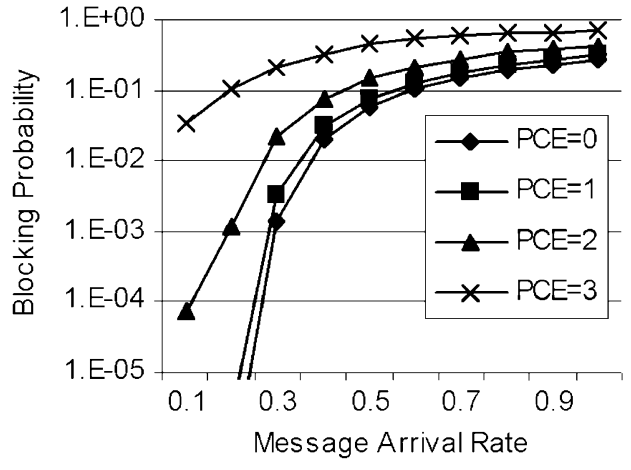

Fig. 9. Effect of PCE on blocking probability (1 LCD, 20 voice).

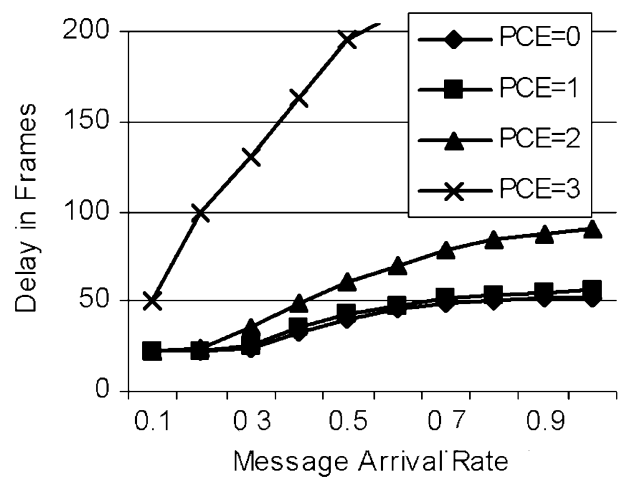

Fig. 10. Effect of PCE on delay (1 LCD, 20 voice) Lin Wang.

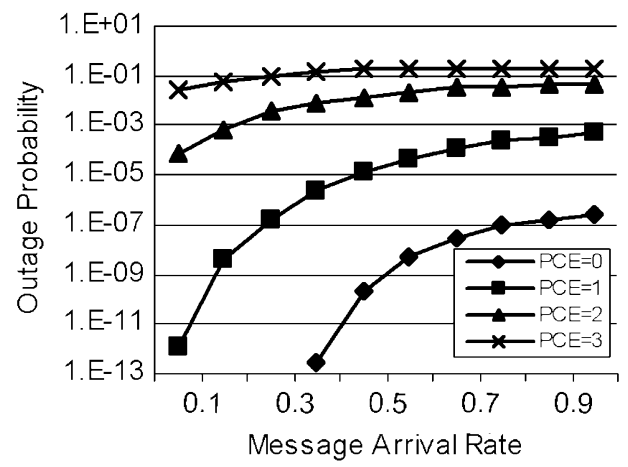

Fig. 11. Outage probability as a function of message rate with various PCEs (1 LCD, 20 voice).

This trend is also reflected in the PS data performance. By comparing overall system performance, including throughput, blocking probability, delay, and outage probability with different PCE values, it is easy to find that, as PCE becomes larger, the performance degradation becomes more serious.

Another interesting point is shown in Fig. 8. Here, the throughput increases as the PS traffic load increases and then starts turning down after a maximum throughput is reached. Moreover, the traffic load associated with the maximum throughput becomes increasingly less as the PCE value becomes larger. This is quite different from the throughput performance without PCE. In that case, throughput continues to increase as PS traffic load increases and then tends to flatten out as shown in Fig. 8. In imperfect power control, the throughput is also subject 
to the outage probability caused by intracell PS users due to their PCEs. The increase in the PS traffic leads to an increase in the number of users in the queue, which, on average, will result in more users simultaneously transmitting their packets $(K 2)$. Without PCE, the increase in the average number of simultaneously transmitting users will lead to a higher throughput.

However with PCE, the increase in the average number of simultaneous transmitting users will cause an increase in outage events, which has a negative effect on throughput. With a light traffic load, the outage probability caused by intracell users' PCEs is rather small, and its negative effect on throughput is compensated by the increase in the number of simultaneous transmitting users [K2 in (15)]. Thus, throughput increases as PS traffic load increases. However, with further increase of PS traffic load, the increase in the number of simultaneous users cannot compensate for the outage probability caused by themselves. Hence, the throughput is brought down. Also, the outage probability becomes larger as PCE increases with the same traffic load. So, with a larger PCE, the negative effect increases faster than the positive effect caused by the increase in the number of simultaneous transmitting users. So, with a large PCE, the PS traffic load associated with the maximum throughput is lower.

With a fixed $T$, the congestion control attempts to keep the number of simultaneously transmitting PS data users below a threshold, so when the load is heavy this number stays close to this threshold. For instance, with perfect power control and with a further increase in traffic load, as in Fig. 9, $\lambda_{d}$ is more than 0.7 for $\mathrm{PCE}=0$, and there is no rapid increase in throughput; hence, the throughput curve tends to be flat. With imperfect power control, because the average number of simultaneously transmitting users is almost constant, the average outage probability is also kept at an almost constant value. Thus, the throughput curves in Fig. 9 for imperfect PCE also become flat, after the PS traffic load is greater than 0.7 , which is the same as for the perfect power control.

\section{CONCLUSION}

Integrated CS/PS cellular systems are studied. An analytical model based on interference modeling is developed for the study. With this analytical model, the effects of congestion control are addressed because with non-real-time PS services, congestion control at link level is one of most popular means to improve system efficiency.

Because intercell and intracell interference interact with each other through the congestion control function, a recursive approach is used to derive the intercell interference. Study of the congestion control function suggests that the control threshold $T$, which determines the tradeoff between CS quality and PS performance, needs to be carefully dealt with in the multicell environment. Moreover, in the model, a Markov chain model is integrated into the interference modeling method so the effects caused by the PS DFT transmission mode, are studied in detail. The investigation is also extended to the system performance with PCEs. The numerical results on system capacity performance show the basic trend of capacity reduction caused by
PCE. In this study, it is found that, compared with the single cell case, capacity loss in a multicell situation decreases as the PCE increases because, as the PCE increases, the outage probability caused by PCE become more and more dominant. Performance of PS in the integrated PS/CS environment is also studied with PCEs. The results show that, when the PCE becomes larger, the degradation in PS performance becomes worse. So, a close and fast power control scheme would also be quite useful for packet transmission.

\section{APPENDIX}

Because $\Gamma_{i}$ is a log-normal random variable, we first approximate $Y_{i}=\left(G_{i} / \Gamma_{i}+1\right)^{-1}$ as a log-normal random variable. The approximation is justified as follows by using the moment match method. Let

$$
E\left[Y_{E}\right]=E\left[Y_{A}\right]
$$

and

$$
D^{2}\left[Y_{E}\right]=D^{2}\left[Y_{A}\right]
$$

where $Y_{E}$ and $Y_{A}$ are the exact and approximated random variables, respectively.

So, with SIR following a log-normal distribution and $Y$ approximated as a log-normal distribution, then we have

$$
\exp \left(-\mu_{\Omega_{i}} \beta+\beta^{2} \sigma_{e}^{2} / 2\right) G_{i}+1=\exp \left(\mu_{Y}+\frac{\sigma_{Y}^{2}}{2}\right)
$$

and

$$
\begin{gathered}
\exp \left(-2 \mu_{\Omega_{i}} \beta+\beta^{2} \sigma_{e}^{2}\right)\left(\exp \left(\beta^{2} \sigma_{e}^{2}\right)-1\right) G_{i}^{2} \\
=\exp \left(2 \mu_{Y}+2 \sigma_{Y}^{2}\right)\left(\exp \left(\sigma_{Y}^{2}\right)-1\right) .
\end{gathered}
$$

Then the $\mu_{Y}$ and $\sigma_{Y}$ can be found as shown in (26) and (27).

Computer simulation results show that the approximation matches the actual value quite well. The Kolmogorov-Smyrnov statistic [15] is found to be less than $0.01,0.017$, and 0.042 for $\sigma_{e}=3,2,1 \mathrm{~dB}$ for voice users, respectively.

Thus, $Y$ 's s Gaussian mean and variance are derived through matching the first and second moments as

$$
\mu_{Y_{i}}=\ln \left(\frac{\exp \left(-\mu_{\Omega_{i}} \beta+\beta^{2} \sigma_{e}^{2} / 2\right) G_{i}+1}{\exp \left(\beta^{2} \sigma_{e}^{2} / 2\right)}\right)
$$

and

$$
\sigma_{Y_{i}}^{2}=\ln \left(\frac{\exp \left(-2 \mu_{\Omega_{i}} \beta+\beta^{2} \sigma_{e}^{2}\right)\left(\exp \left(\beta^{2} \sigma_{e}^{2}\right)-1\right) G_{i}^{2}}{\left(\exp \left(-\beta \mu_{\Omega_{i}}+\beta^{2} \sigma_{e}^{2} / 2\right) G_{i}+1\right)^{2}+1}\right)
$$

where $\beta=(\ln (10) / 10)$.

Then as a sum of the log-normal random variables $Y, Z$ is well approximated as a log-normal random variable $e^{X_{z}}$ [16], where $X_{z}$ is a Gaussian random variable, with mean $m_{z}(k)=$ 


$$
\begin{aligned}
& \ln \left(\sum_{i=1}^{k} \exp \left(\mu_{Y_{i}}+\left(\sigma_{Y_{i}}^{2} / 2\right)\right)\right)-\left(\sigma_{z}^{2} / 2\right) \text { and variance } \\
& \sigma_{z}^{2}(k)=\ln \left(\frac{\sum_{i=1}^{k} \exp \left(2 \mu_{Y_{i}}+\sigma_{Y_{i}}^{2}\right)\left(\exp \left(\sigma_{Y_{i}}^{2}\right)-1\right)}{\left(\sum_{i=1}^{k} \exp \left(\mu_{Y_{i}}+\frac{\sigma_{Y_{i}}^{2}}{2}\right)\right)^{2}}+1\right) .
\end{aligned}
$$

\section{ACKNOWLEDGMENT}

The authors would like to thank our colleagues within the EVEREST project for their valuable comments.

\section{REFERENCES}

[1] K. S. Gilhousen, I. M. Jacobs, R. Padovani, A. J. Viterbi, and L. A. Weaver, "On the capacity of a cellular CDMA system," IEEE Trans. Veh. Technol., vol. 40, pp. 303-312, May 1991.

[2] M. G. Jansen and R. Prasad, "Capacity, throughput, and delay analysis of a cellular DS-CDMA system with imperfect power control and imperfect sectorization," IEEE Trans. Veh. Technol., vol. 44, pp. 67-75, Feb. 1995.

[3] F. D. Priscoli and F. Sestini, "Effects of imperfect power control and user mobility on a CDMA cellular network," IEEE J-SAC, vol. 14, no. 9, pp. 1809-1817, Dec. 1996.

[4] E. Kudoh, "On the capacity of DS/CDMA cellular mobile radios under imperfect transmitter power control," IEICE Trans. Commun., vol. E76-B, no. 8, pp. 886-892, Aug. 1993.

[5] B. Hashem and E. S. Sousa, "Reverse-link capacity and interference statistics of a fixed-step power-controlled DS/CDMA system under slow multi-path fading," IEEE Trans. Commun., vol. 47, no. 12, pp. 1905-1912, 1999.

[6] D. K. Kim and D. K. Sung, "Capacity estimation for an SIR-based powercontrolled CDMA system supporting ON-OFF traffic," IEEE Trans. Veh. Technol., vol. 49, no. 7, pp. 1094-1101, 2000.

[7] S. Manji and W. Zhuang, "Power control and capacity analysis for a packetized indoor multimedia DS-CDMA network," IEEE Trans. Veh. Technol., vol. 49, no. 3, pp. 67-74, May 2000.

[8] D. K. Kim and F. Adachi, "Theoretical analysis of reverse link capacity for an SIR-based power-controlled cellular CDMA system in a multipath fading environment," IEEE Trans. Veh. Technol., vol. 50, no. 2, pp. 452 464, Mar. 2001.

[9] A. J. Viterbi, CDMA: Principles of Spread Spectrum Communications, 2nd ed., Reading, MA: Addison-Wesley, 1995.

[10] S. Ariyavisitakul, "Signal and interference statistic of a CDMA system with feedback power control-Part II," IEEE Trans. Commun., vol. 42, no. 2-4, pp. 597-605, 1994.

[11] Services Provided by the Physical Layer [Online]. Available: http:// www.3gpp.org/.

[12] L. Kelinrock, Queueing Systems—Volume I: Theory. New York: Wiley, 1976.

[13] Simulation Tools: Inherited Features and Newly Implemented Capabilities [Online]. Available: http://www.everest-ist.upc.es. IST-2002-001858: EVEREST, D07.

[14] S. J. Oh and K. M. Wasserman, "Dynamic spreading gain control in multiservice CDMA networks," IEEE J-SAC., vol. 17, no. 5, pp. 918-917, May 1999.

[15] W. H. Press, S. A. Teskolsky, W. T. Vetterling, and B. P. Flannery, Numerical Recipes in C, 2nd ed., Cambridge, MA: Cambridge University Press, 1992.

[16] F. N. Fenton, "The sum of lognormal probability distributions in scatter transmission systems," IRE Trans. Commun. Syst., vol. CS-8, no. 3, pp. 5767, 1960.

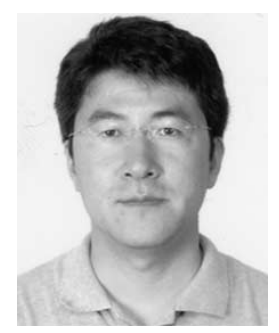

Lin Wang received the B.S. degree in radio physics from Xiamen University, Xiamen, China, in 1997. He received the Ph.D. degree in telecommunication from King's College, London University, London, England, in 2003.

In 1998, he joined the Centre for Telecommunications Research, King's College London, London, England, as a research student. From 2000 to 2003, he worked as a Research Associate on the Engineering and Physical Sciences Research Council-sponsored project, "Advanced MAC Strategies on Future W-
CDMA Packet Networks." Currently, he is working as a Senior Research Fellow on the European Union-funded project EVEREST to develop advanced radio resource algorithms for third-generation $(3 \mathrm{G})$ and beyond $3 \mathrm{G}$ systems.

Hamid Aghvami (F'04) joined the academic staff at King's College, London, England, in 1984. In 1989, he was promoted to Reader and Professor in Telecommunications Engineering in 1992. Currently, he is the Director of the Centre for Telecommunications Research at King's College. He serves as a consultant on digital radio communications systems for British and international companies. He was Visiting Professor at NTT Radio Communication Systems Laboratories in 1990 and Senior Research Fellow at BT Laboratories from 1998 to 1999. He is currently an Executive Advisor to Wireless Facilities, Inc., and Managing Director of Wireless Multimedia Communications LTD, London, UK (his own consultancy company). He leads an active research team working on numerous mobile and personal communications projects for third- and fourth-generation systems; these projects are supported both by the government and by private industry. He has published more than 300 technical papers. He has given invited talks on various aspects of personal and mobile radio communications and taught courses on the subject worldwide.

Dr. Aghvami is a distinguished lecturer and a member of the Board of Governors of the IEEE Communications Society. He has been a member, Chairman, and Vice-Chairman of the technical program and organizing committees of a large number of international conferences. He is also founder of the International Conference on Personal Indoor and Mobile Radio Communications (PIMRC) $\mathrm{He}$ is a fellow of the Royal Academy of Engineering and a fellow member of the IEEE, 2004.

Williams G. Chambers was born in Leeds, England, in 1937. He received the B.Sc. degree in mathematics from the University of Cambridge, Cambridge, England, in 1959, and the Ph.D. degree from University of Cambridge, Cambridge, UK, in 1963

He was a lecturer in mathematics at Westfield College and at Royal Holloway and Bedford New College, both in the University of London, London, England, until his transfer to the Electronic Engineering Department at King's College London, London, England, in 1986. He has worked in a number of fields, including solid-state physics and Fourier transform spectroscopy. Currently, his main interests are in coding theory, cryptology, chaos theory, and spread-spectrum communications. He is the author of a book Basics of Communications and Coding (Oxford, England: Oxford University Press, 1985) and of more than 40 articles in refereed journals.

Jordi Pérez-Romero is Assistant Professor in the field of radio communications in the Radio Communications Group, Signal Theory and Communications Department, Universitat Politècnica de Catalunya (UPC), Barcelona, Spain. He has been involved in different European Projects and in projects for private companies. His main research areas are in the field of radio resource management strategies for third-generation and heterogeneous networks and in packet transmission mechanisms for CDMA mobile communications. He has published several papers on these topics in IEEE journals and conferences.

Oriol Sallent is Associate Professor at the Universitat Politènica de Catalunya (UPC), Barcelona, Spain. His research interests are in the field of mobile communication systems, especially packet radio techniques, spread-spectrum systems, radio resource, and quality-of-service management. He has published several papers on these topics in IEEE journals and conferences. He has participated in many research projects and consultancies funded by either public organizations or private companies.

Dr. Sallent received the Doctorate Award from the Telecommunication Engineer Association of Spain in 1997 for his Ph.D. dissertation on multiple access protocols for CDMA-based systems. 
Mischa Dohler (M'03) obtained the M.S. degree in telecommunications from King's College, University of London, London, England, in 1999; the Diploma in electrical engineering from Dresden University of Technology, Dresden, Germany, in 2000; and the Ph.D. degree from King's College London, London, England, in 2003.

He is currently a Lecturer at the Centre for Telecommunications Research, King's College London. His current research interest lies in distributed-MIMO multistage ad hoc and mesh networks and related areas. Prior to telecommunications, he studied physics in Moscow, Russia. He won various competitions in mathematics and physics and participated in the third round of the International Physics Olympics for Germany. He has published numerous research papers and holds four patents. He is a Student Representative of the IEEE UKRI Section and a member of the Student Activity Committee of IEEE Region 8. He is a London Technology Network business fellow for King's College London. 